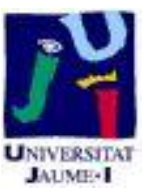

Título artículo / Títol article: High submicellar liquid chromatography

Autores / Autors

Ruiz Ángel, M. J. ; Carda Broch, Samuel ; García

Álvarez-Coque, M. C.

Revista:

Separation \& Purification Reviews Volume 43, Issue 2, 2014

Versión / Versió:

Preprint de l'autor

Cita bibliográfica / Cita bibliogràfica (ISO 690):
RUIZ-ÁNGEL, Maria Jose; CARDA-BROCH, Samuel; GARCÍA-ÁLVAREZ-COQUE, Maria Celia. High Submicellar Liquid Chromatography. Separation \& Purification Reviews, 2014, 43.2: 124-154.

http://hdl.handle.net/10234/125069 


\title{
High submicellar liquid chromatography
}

\author{
M.J. Ruiz-Ángel, ${ }^{\mathrm{a}}$ S. Carda-Broch, ${ }^{\mathrm{b}}$ M.C. García-Álvarez-Coque ${ }^{\mathrm{a} *}$ \\ ${ }^{a}$ Departament de Química Analítica, Universitat de València, c/Dr. Moliner 50, Burjassot (Spain) \\ ${ }^{b}$ Departament de Química Física i Analítica, Universitat Jaume I, Cra. Borriol s/n, Castelló (Spain)
}

\section{Abstract}

In 1980, the addition of a surfactant above the critical micellar concentration (CMC) in reversed-phase liquid chromatography (RPLC) was proposed as a way to modify the selectivity and analysis time. This gave rise to a new chromatographic mode which was called micellar liquid chromatography (MLC). However, with conventional columns, solutions containing only surfactant were too weak and yielded poor peak shape. This was remediated by the addition of a small amount of organic solvent to the pure micellar mobile phase. Since then, in order to preserve the existence of micelles, analysts working in MLC avoid usually high amounts of organic solvent in the mobile phase. Nevertheless, there is no reason to neglect the potentiality of mobile phases containing a surfactant above its $\mathrm{CMC}$ in water and a high concentration of organic solvent, where micelles cannot be formed (submicellar conditions). This chromatographic mode has been called high submicellar liquid chromatography (HSLC), and can be considered as a bridge between MLC and conventional RPLC. There is no sudden breakdown of micelles with addition of organic solvent, and accordingly, the transition between MLC and HSLC is easily not noticeably. For this reason, in the literature, some authors have claimed to be working in MLC conditions, without being aware that no micelles were formed. The combination of stronger elution strength, larger selectivity and improved peak shape, with respect to MLC and conventional RPLC, makes HSCL a promising chromatographic mode, which achieves in practical times separations of compounds unresolved, or highly retained with other RPLC modes. This work offers some insights on the interactions that occur inside the chromatographic column, the modification of the stationary and mobile phases, modelling of retention, peak shape implications, and separation performance in HSLC, in comparison to MLC and conventional RPLC.

Keywords: Reversed-phase liquid chromatography; Surfactant-mediated chromatographic modes; Submicellar liquid chromatography; Column interactions; Chromatographic performance

\footnotetext{
* Corresponding author: Tel.: +34 96354 4014; fax: +34 963544436. e-mail address: celia.garcia@uv.es (M.C. García-Álvarez-Coque)
} 


\section{Introduction}

The addition of surfactants to a hydro-organic mobile phase in reversed-phase liquid chromatography (RPLC) produces significant changes in the chromatographic behaviour [1-3]. Particularly interesting is the use of ionic surfactants, such as sodium dodecyl sulphate (SDS) and cetyltrimethylammonium bromide (CTAB), in the analysis of compounds bearing an opposite charge. The surfactants are significantly adsorbed on the surface of the non-polar stationary phase (usually of the bonded alkylsilica gel type), creating a charged asymmetric bilayer, which acts as a dynamic ion-exchanger for ionic analytes. Oppositely charged solute ions are attracted by the adsorbed surfactant ions and yield high retention, whereas solute ions with similar charges as the surfactant are repulsed and elute with the void volume.

When the surfactant is added at low concentration (below the critical micellar concentration, CMC), the stationary phase is progressively coated with surfactant, and the mobile phase is comprised of a rather small amount of free monomers (Fig. 1a). Above the CMC, the column reaches saturation or only small changes are produced in the surfactant coating depending on the column and surfactant nature [4]. However, the mobile phase experiences major changes, since the surfactant monomers aggregate to form small clusters or micelles (with the non-polar hydrocarbon chain in the monomers oriented towards the micelle core, and the neutral or ionic head towards its surface) (Fig. 1b and d). The mobile phase may contain only water, buffer and micelles, which play the role of an organic modifier. Micelles notably modify the solubility and transference of solutes between mobile phase and stationary phase, which have particular implications with regard to selectivity, analysis time and efficiency, as first demonstrated by Armstrong and Henry in 1980 [5]. Owing to the presence of micelles, this chromatographic mode was named pseudophase liquid chromatography or micellar liquid chromatography (MLC). In this mode, solute separation is achieved on the basis of the differential partitioning between bulk solvent and monomers of surfactant coating 
the stationary phase, and between bulk solvent and micelles. However, with conventional columns, solutions containing only surfactant are too weak and yield poor peak shape. For this reason, the addition of a small amount of organic solvent to the mobile phase was soon suggested to enhance the elution strength and efficiency $[1,6]$.

The influence on the retention of the organic solvent (below or above the CMC) is similar to that for systems without surfactant: the retention times of analytes decrease as a result of the decreased polarity of the bulk solvent. However, an additional reduction in the retention is produced by the competition between organic solvent molecules and surfactant monomers for adsorption sites, which reduces the amount of surfactant adsorbed on the stationary phase [7]. Concomitantly, the efficiency is enhanced due to the thinner surfactant coating. Such is the improvement in chromatographic performance in the presence of an organic solvent that most analyses in MLC are performed with mobile phases containing both surfactant and organic solvent $[1,8]$.

The presence of surfactant in the mobile phase allows the use of organic solvents scarcely miscible with water, reaching concentrations useful in RPLC. However, in spite of the wide range of compatible solvents, only the aliphatic alcohols 1-propanol, 1-butanol and 1-pentanol are routinely used in MLC to develop analytical methods, being 1-propanol the most common. Surprisingly, there are only few reports with acetonitrile, which is the solvent of choice in conventional RPLC. Some authors have recommended the use of methanol or ethanol, but their elution strength in the presence of surfactant is rather weak.

In MLC, the concentration of organic solvent is limited to preserve the integrity of micelles. At high concentrations of organic solvent, micelles breakdown (i.e. disaggregate) [9]. In this situation, interactions between analytes and free surfactant monomers (instead of micelles) will coexist in the bulk solvent with interactions with the still surfactant-modified stationary phase (Fig. 1c). This gives rise to a new chromatographic mode inbetween MLC 
and conventional RPLC, which is the topic of this review [2,10], which has been called high submicellar liquid chromatography (HSLC).

Consequently, depending on the concentration level of surfactant and organic solvent, four RPLC modes (with transition regions) are possible, each with particular performances (Fig. 2):

(i) Hydro-organic (conventional) RPLC without additives other than the buffer components.

(ii) Ion-pair chromatography (IPC), where the concentration of surfactant (an ionic surfactant) is below the CMC, and the organic solvent content is similar or larger than the usual in conventional RPLC. The mobile phase contains a rather small amount of surfactant monomers (Fig. 1a). Since there is a pre-micellization situation, this chromatographic mode has been also called submicellar liquid chromatography, which was a rather common name in the first reports in MLC [11].

(iii) Micellar-liquid chromatography, where the mobile phase contains a rather small amount of monomers of surfactant (ionic or non-ionic), and pure micelles (without organic solvent), or hybrid micelles (built with surfactant monomers and organic solvent molecules). In the latter case, micelles are in a hydro-organic environment (Fig. 1b).

(iv) High submicellar liquid chromatography (HSLC), where the surfactant (ionic or non-ionic) is at a concentration where micelles are formed in water, and the organic solvent content is high. This prevents the formation of micelles: only surfactant monomers exist in the mobile phase (a rather large amount), which are dissolved in a hydro-organic medium (Fig. 1c). The name HSLC refers to a quantitative difference with IPC (submicellar liquid chromatography) with respect to the concentration of surfactant monomers in the mobile phase, which gives rise to a particular behaviour.

This work is concerned with the main features of HSLC. The chromatographic performance under micelle breakdown conditions in the mobile phase has still been scarcely 
studied. However, some attractive advantages have been observed with respect to MLC and hydro-organic RPLC, in terms of analysis time, selectivity and peak shape, which deserve some attention [2,10,12-15]. This review tries to put forward a unifying description of the behaviour of HSLC among the surfactant-mediated chromatographic modes.

\section{Solute interactions with stationary phase and mobile phase}

In IPC, an ion-exchange retention mechanism with the stationary phase is dominant and the interaction with the ionic surfactant in the mobile phase is rather small (Fig. 1a) [16]. In MLC, both ionic and non-ionic surfactants are used, with SDS by far the most common $[1,8]$. The stationary phase reaches saturation and the interaction of solutes with the adsorbed surfactant is again the prevalent equilibrium [1]. However, a secondary equilibrium is established between solutes and micelles in the mobile phase, which is affected by the presence of organic solvent (Fig. 1b) [17]. In general, the retention increases with respect to conventional RPLC, but in a smaller amount compared to IPC at similar organic solvent contents due to the presence of micelles in the mobile phase, which enhance the solubilization capability [18]. As long as a certain amount of surfactant remains adsorbed and micelles exist, the retention mechanism will be the typical of the micellar mode. Finally, in HSLC, where micelle disaggregation occurs, the retention mechanism will depend on the amount of surfactant that still remains adsorbed on the alkyl-bonded phase, and on the interaction of solutes with surfactant monomers in the bulk mobile phase, which replace the micelles (Fig. 1c). It can be expected that, without surfactant adsorption, the observed effect on the retention and resolution of analytes would be solely a result of the interactions with the surfactant monomers in the mobile phase, in addition to the interaction with the non-polar bonded phase and organic solvent. 
The adsorption of a surfactant onto the stationary phase can occur in at least two ways:

(i) The long alkyl or polyoxypropylene chains of the surfactant would interact with the alkyl bonded chains on the stationary phase and the hydrophilic head groups would stick out in contact with the polar solution, as revealed by nuclear magnetic resonance (NMR) studies [19], giving rise to an open micelle-like structure $[20,21]$. This is the situation that is expected with SDS and C18 bonded silica (Fig. 1a-c).

(ii) In the case of cationic surfactants, the ionic head group can be strongly adsorbed, the stationary phase would behave then as a more hydrophobic surface. This is very likely the case of CTAB adsorbed on a silica surface, which gives rise an RPLC mode [22] (Fig. 1d).

In the case of an anionic surfactant, such as SDS, the negatively charged asymmetric bilayer with SDS affects the penetration depth of solutes into the bonded phase [23,24]. The adsorbed surfactant monomers attract strongly cationic basic drugs increasing their retention, as a consequence of a combination of electrostatic (with the anionic sulphate group) and hydrophobic (with the uncovered alkyl-bonded layer and surfactant chain) interactions, the latter being weaker compared to a hydro-organic system [3].

\section{Micellar or submicellar conditions?}

\subsection{Micelle breakdown in the presence of organic solvent}

The organic solvent molecules can bind the micelles and modify their shape. Short-chain alcohols (ethanol and propanol) interact with the micelle surface, reducing the repulsions among the ionic heads of the surfactant monomers forming the micelle, whereas more hydrophobic alcohols (butanol and pentanol) are inserted into the non-polar micelle. This affects micelle formation and the partitioning equilibria $[25,26]$. 
Organic solvent molecules can prevent the formation of micelles [9,26]. Therefore, the amount of organic solvent that can be added in MLC is limited not only by its solubility, but also by micelle disaggregation: depending on the nature of the surfactant and organic solvent, there is a limiting concentration of organic solvent above which micelles do not occur anymore. Thus, for example, it is well accepted that SDS micelles are disrupted at concentrations (v/v) above 30-40\% methanol, 30\% ethanol, 22\% 1-propanol and 30\% acetonitrile, although these values are not conclusive [9]. Also, CTAB micelles do not exist in solutions with more than $20 \%$ methanol [11]. It should be also noted that because of their geometry, some surfactants, such as tetraheptylammonium bromide (THPA), are not able to form micelles at any condition [25].

The accurate evaluation of micelle disaggregation at increasing organic solvent contents is problematic, since the transition to a situation where micelles do not exist is gradual (there is no sudden breakdown of micelles), with a progressive reduction in the aggregation number [27]. This fact, and the absence of remarkable changes in the chromatographic behaviour when micelles breakdown, do not allow knowing exactly if micelles still exist. It is thus not surprising that, in the literature, some authors using high concentrations of organic solvent have claimed to be working in MLC conditions, without being aware that no micelles were formed (Table 1) [28-35]. These reports correspond thus to the HSLC mode.

\subsection{Determination of the critical micellar concentration}

In the presence of organic solvent, micelle parameters, such as the CMC, are altered. Thus, in order to know the existence of micelles, it is convenient to determine the CMC value at different conditions. For an ionic surfactant, this can be easily carried out through a conductimetric titration. For this purpose, the surfactant is added below and above the CMC. Then, the intersection of the straight-lines fitted in each region representing the conductance 
versus the surfactant concentration is obtained after the needed corrections for changing volumes [36]. Micelle modification can be also monitored by following changes in the surface tension of surfactant solutions. The drop weight procedure is based on the influence of this property on the size of a drop formed when the liquid is suspended from a glass tip. Information about micelles can be provided by weighting a given amount of drops delivered from a burette at changing surfactant concentration [2].

The changes in the drop weight for SDS upon addition of an organic solvent have been observed to correlate with the changes in retention and peak shape for basic compounds [2]. This also revealed some micelle perturbation and possible disaggregation. Thus, the drop weight was observed to remain approximately constant in the 5-15\% acetonitrile range, followed by a gradual decrease in the $20-40 \%$ range (with $0.075-0.15$ M SDS). In the same report, the change in the retention behaviour indicated that above $20 \%$ acetonitrile, the micelle structure was significantly altered, with a likely breakdown ca. 30\% acetonitrile. The addition of 1-propanol instead of acetonitrile to an SDS micellar solution resulted in a different behaviour: the drop weight decreased up to 20-25\% 1-propanol. Also, it did not depend on the surfactant concentration above 15\% 1-propanol [27].

There is much work on the determination of the CMC for SDS, whose values in water are in the range 8.2-8.4 $\mathrm{mM}$ [1]. This value increases upon addition of both acetonitrile and methanol (e.g. at $20 \%$ acetonitrile, it is $\sim 30 \mathrm{mM}$ ), while decreases in the presence of the less polar longer alcohols (ethanol, 1-propanol, 1-butanol and 1-pentanol), with an increasing rate depending on the chain length [26]. This means that the surfactant distribution is shifted towards the bulk solvent or the micelles, respectively. However, the effects of the addition of organic solvent to a micellar mobile phase is not very dramatic until a concentration of the organic solvent is reached where micelles disaggregate, so that the micellar phase is converted into a submicellar phase. 
In the presence of 1-propanol, the CMC for SDS was estimated to be: 7.2, 6.3, 6.0 and $5.9 \mathrm{mM}$ in 1.5, 3.0, 4.5 and 6\% 1-propanol [37], and 5.75, 4.65, 3.4 and $2.7 \mathrm{mM}$ in 5, 10, 15 and 20\% 1-propanol, respectively [26]. A wide compilation of CMC values for different types of surfactants can be found in Ref. [1].

\section{Surfactant adsorption}

\subsection{Stationary phase saturation by surfactant in aqueous medium}

The adsorbed amount of surfactant on an RPLC column increases rapidly and reaches a plateau above a certain concentration of surfactant. Berthod et al. [38-40] reported adsorption isotherms for SDS and CTAB on five Hypersil stationary phases of various polarities: three apolar silica (methyl bonded silica SAS, octyl bonded silica MOS, and octadecyl bonded silica ODS), and two polar silica (cyanopropyl bonded silica CPS and naked silica). These authors found that the adsorbed amount of surfactant for a saturated column was similar on alkyl-bonded silica $(\mathrm{C} 1, \mathrm{C} 8$, and $\mathrm{C} 18$, with the $\mathrm{C} 1$ phase adsorbing the largest amount instead of the more hydrophobic C18 phase) $\left(4.0-5.0 \times 10^{-6} \mathrm{~mol} / \mathrm{m}^{2}\right)$, and was only $2.5 \times 10^{-6} \mathrm{~mol} / \mathrm{m}^{2}$ of SDS and $3.5 \times 10^{-6} \mathrm{~mol} / \mathrm{m}^{2}$ of CTAB on CPS Hypersil, and $0.5 \times 10^{-6} \mathrm{~mol} / \mathrm{m}^{2}$ of SDS and $2.0 \times 10^{-6} \mathrm{~mol} / \mathrm{m}^{2}$ of $\mathrm{CTAB}$ on naked silica. The higher adsorbed amount for CTAB with respect to SDS was explained by the attraction of the CTAB cations to the ionized silanol groups, whereas the higher amount of adsorbed surfactant for the $\mathrm{C} 1$ bonded phase was attributed to mixed polar and hydrophobic interactions, confirming the assumption that hydrophobic interactions are not the only ones responsible for surfactant adsorption. The collapse of hydrocarbon moieties of long alkyl bonded phases on the surface, in the presence of water (which reduces the number of sites for hydrophobic interactions [41]), has also been suggested as the reason for the low surfactant adsorption on the $\mathrm{C} 18$ silica as compared to $\mathrm{C} 1$ bonded silica. 
Maximal adsorbed SDS was found close to one surfactant molecule per bonded moiety for $\mathrm{C} 1$ and $\mathrm{C} 8$ grafted phases, and close to two SDS molecules for a C18 phase [40]. Examination of the hysteresis loop for a C18 material modified with surfactant provided additional information about the extent of stationary phase modification [24,42]. The BET surface area (surface available to the nitrogen molecules at $77 \mathrm{~K}$ ) was found to decrease about $60 \%$ for both non-ionic and anionic surfactants (Brij-35 and SDS, respectively). The general pore shapes of the parent C18 material appeared to be retained with the surfactant-modified material. The adsorbed surfactant molecules seem to fill part of the silica pore volume, producing a thick continuous film on the interior walls, rather than completely filling the pores. On doing so, the stationary phase surface area is reduced.

There is some disagreement on the conditions needed to reach stationary phase saturation by surfactant. Some authors have found a constant amount of adsorbed surfactant for ionic surfactants above the CMC $[36,43]$. This has been explained by the fact that the concentration of free surfactant monomers in bulk solution is constant and equal to the CMC, whereas only the concentration of micelles increases as the total surfactant concentration is raised (and the micelles are not adsorbed). In contrast, according to Berthod et al., the assumption that the column is saturated with surfactant above the CMC seems to be not the case for all surfactant/stationary phase combinations [38,39]. Surfactant adsorption may continue with an increase as much as $20 \%$ of the total sorption at surfactant concentrations greater than twice the CMC. In fact, a plateau with constant adsorbed amount for SDS was only observed with C18 Hypersil.

\subsection{Adsorption isotherms}

When the concentration of an analyte in the solution in contact with the stationary phase (i.e. the adsorbent) is being increased, its concentration on the adsorbent steeply rises at first, 
but then the rate of increase of the adsorbed amount gradually diminishes as it approaches the maximal capacity of the adsorbent (i.e. its saturation). This behaviour in RPLC systems is most often described by the Langmuir isotherm, or by models departing from it [44]:

$Q=\frac{a c}{1+b c}$

where $Q$ and $c$ are the analyte concentration in the stationary phase and mobile phase, respectively, $a$ is the distribution coefficient at infinite dilution, and $b$ is related to the saturation capacity of the adsorbent, $Q_{\mathrm{s}}=a / b$. According to the Langmuir isotherm, this should be achieved at infinite analyte concentration in the mobile phase. However, experimental adsorption isotherms for surfactants in RPLC systems showed profiles different from the isotherms of simple organic compounds. The isotherms presented an abrupt break, and the adsorbed amount remained approximately constant above a certain value of the surfactant concentration in bulk solution equal or higher than the CMC [38,39].

Jandera and Fischer modified the Langmuir isotherm to describe the distribution of surfactants between stationary phase and mobile phase in the submicellar concentration range [36]. The maximal adsorbed concentration was assumed to correspond to $c_{\mathrm{s}}=\mathrm{CMC}$ :

$Q=\frac{a^{\prime} c_{\mathrm{s}}}{1+b^{\prime} c_{\mathrm{s}}}=\frac{a^{\prime} c_{\mathrm{s}}}{1+\left[\frac{a^{\prime}}{Q_{\mathrm{CMC}}}-\frac{1}{\mathrm{CMC}}\right] c_{\mathrm{s}}}$

where $a^{\prime}$ and $b^{\prime}$ are constants for the modified Langmuir isotherm. This equation applies only for $c_{\mathrm{s}}<\mathrm{CMC}$, whereas $Q=Q_{\mathrm{CMC}}$ (the amount adsorbed at the plateau) for $c_{\mathrm{s}} \geq \mathrm{CMC}$. Eq. (2) should be taken as a first approximation, since as indicated, surfactant adsorption may continue above the CMC. 


\subsection{Removal of surfactant from the stationary phase in the presence of an organic solvent}

In surfactant-mediated chromatographic systems, the surfactant modifies the stationary phase by coating it totally or partially. The organic solvent added to the mobile phase reduces the coating thickness, which depends on the surfactant/organic solvent ratio. In fact, moderate amounts of alcohols added to an SDS micellar mobile phase have been found to reduce significantly the amount of adsorbed surfactant, with a clear trend that depends on the molecular weight of the alcohol (i.e. its hydrophobicity): while the addition of 5\% methanol reduced the amount of SDS by ca. 10\%, 5\% 1-pentanol reduced it by ca. 50\% [24]. The influence of methanol was found to be more significant with the anionic SDS than with the cationic CTAB and Septonex (carbethoxypentadecyl trimethyl ammonium bromide) [11].

In addition to reducing the coating thickness (and consequently, the carbon loading), the addition of alcohols is also expected to influence the fluidity/rigidity of the surfactant/C18 bonded ligand structure, just as their presence alters the fluidity of the micellar aggregate structure in the mobile phase [42]. This should improve the efficiency, since the solute diffusion coefficient ought to increase as the microviscosity of the phase decreases. In the limit, the BET surface area, the cumulative pore volume and chromatographic efficiency will reapproach that of the unmodified $\mathrm{C} 18$ stationary phase.

Berthod and Roussel reported a linear decrease in the adsorbed amount of SDS upon addition of several organic solvents, including methanol and 1-propanol [40]. The desorption rate of SDS for methanol was 9-folded smaller compared to 1-propanol. The maximal concentration of both modifiers examined by these authors was 5\% methanol and 3\% 1-propanol. If the linear patterns were followed at larger concentrations, the surfactant would be completely desorbed for $95 \%$ methanol and 10\% 1-propanol. However, the assumption of a linear decrease of adsorbed surfactant with increasing alcohol contents beyond the studied range is questionable. 
Li and Fritz carried out experiments with several non-ionic surfactants, such as Tween 60 and Pluronic L-31, and ionic surfactants, such as the anionic SDS and DOSS (dioctyisulfosuccinate), and the cationic CTAB or THPA, in the presence of $60 \%$ acetonitrile [10]. The non-ionic surfactants contained alternating hydrophobic polyoxypropylene and hydrophilic polyoxyethylene segments, and the ionic ones were amphiphilic compounds with one or more long alkyl hydrophobic chains and a hydrophilic head group varying in chemical nature. The authors concluded that significant surface adsorption did not occur in the presence of $60 \%$ acetonitrile, by observing that there was no lost of surfactant from the solution, and no column re-equilibration time was needed to obtain a stable baseline.

More recently, the adsorption of SDS on a C18 column, in the presence of up to $50 \%$ acetonitrile was indirectly demonstrated based on the interaction of cationic basic compounds ( $\beta$-blockers) with the free silanols on the column [14]. When SDS is added to the mobile phase, the free surfactant monomers bound to the $\mathrm{C} 18$ chains mask the free silanols on the siliceous support that are the origin of the poor efficiencies and tailing peaks for basic compounds in hydro-organic RPLC with conventional columns [45]. Meanwhile, the stationary phase adopts a negative charge that attracts the cationic solutes. This attraction increases so remarkably the retention times, that for relatively low polar $\beta$-blockers, these are easily beyond $100 \mathrm{~min}$ in mobile phases containing only the surfactant (a significantly larger retention than in conventional RPLC with hydro-organic mixtures) [2]. The improvement in peak shape and the increased retention confirm the coating of the stationary phase by the anionic surfactant.

A comparison of the relative effect of different alcohols in the SDS micellar mobile phases on the retention, elution strength and peak shape for a set of $\beta$-blockers was observed to parallel their ability to desorb SDS surfactant molecules from a C18 bonded stationary phase [27]. The long retention times and high efficiencies found with a C18 Kromasil column 
and mobile phases containing SDS and 50-60\% methanol suggested that a significant amount of surfactant still covered the stationary phase, and for 35\% 1-propanol, the surfactant layer was not either desorbed totally. This agrees with a previous observation on the tight insertion of the surfactant alkyl-chains in the alkyl moieties of the bonded layer of the densely grafted phases [46].

\section{Modelling the retention}

Modelling the retention gives some insight on the chromatographic behaviour of solutes. It is also useful to predict the retention and optimize the separation conditions. Next, the behaviour for HSLC is compared to that observed for conventional RPLC and MLC.

\subsection{Effect of the organic solvent on the chromatographic behaviour}

In conventional RPLC, the elution behaviour is classically modelled as a quadratic relationship between the logarithm of the retention factor $(\log k)$, and the volume fraction of organic solvent in the hydro-organic mixture $(\varphi)$.

$\log k=c_{0}+c_{1} \varphi+c_{11} \varphi^{2}$

where $c_{0}, c_{1}$ and $c_{11}$ are regression coefficients with characteristic values for a given solute and column/solvent system, being $c_{0}$ the logarithm of the retention factor in water. The sign of $c_{1}$ is negative, since the retention decreases as the concentration of organic modifier increases.

The dependence of the retention factor on the concentration of organic solvent in MLC and HSLC can be described by the same form of equation. Thus, for SDS and methanol, and 10-30\% methanol where micelles are present, or 40-60\% methanol where micelles do not exist, the plots were found to be almost linear, although with different slopes [13]. 


\subsection{Three-phase equilibrium model in surfactant-mediated systems}

In MLC, a three-phase equilibrium model relating the retention factor to the concentration of micelles has been proposed (Figs. 1b and d) [47]. For an aqueous micellar solution, a convenient way to describe the retention is the following [48]:

$\frac{1}{k}=\frac{1+K_{\mathrm{AM}}[M]}{K_{\mathrm{AS}}}=\frac{1}{K_{\mathrm{AS}}}+\frac{K_{\mathrm{AM}}}{K_{\mathrm{AS}}}[M]=c_{0}+c_{1}[M]$

where $[M]$ is the concentration of surfactant monomers involved in micelle formation (i.e. surfactant concentration minus the $\mathrm{CMC}$ ), and $K_{\mathrm{AS}}$ and $K_{\mathrm{AM}}$ are the association constants between solute and stationary phase, and solute and micelles, respectively; $c_{0}$ and $c_{1}$ are regression coefficients. The accuracy of Eq. (4) has been widely verified, with experimental errors usually below $2 \%$. This equation is also valid at fixed organic solvent content. Li and Fritz proposed Eq. (4) to describe the retention behaviour in HSLC at constant organic solvent, as a function of the concentration of free surfactant monomers ([S]), instead of the micellized surfactant [10].

In agreement with Eq. (4), the retention of neutral solutes and solutes with a charge opposite to that of the surfactant decreases as $[M]$ (or $[S]$ ) increases, if the analytes are associated to the micelles (or surfactant monomers). On the other hand, the association of solutes with the stationary phase decreases with the percentage of organic solvent in the mobile phase, which should be explained (at least partially), by the reduction of the surfactant layer on the stationary phase. With SDS, the retention factors decrease upon the addition of organic solvents in the order: methanol < acetonitrile < ethanol $<1$-propanol, which correlates with the extent of surfactant desorption from the stationary phase (stronger for 1-propanol) [7].

In MLC with hybrid mobile phases (containing surfactant and organic solvent), the mechanistic model (Eq. (4)) can be reformulated as [48]: 


$$
\frac{1}{k}=\frac{1+K_{\mathrm{AM}}[M] \frac{1+K_{\mathrm{MD}} \varphi}{1+K_{\mathrm{AD}} \varphi}}{K_{\mathrm{AS}} \frac{1+K_{\mathrm{SD}} \varphi}{1+K_{\mathrm{AD}} \varphi}}
$$

where $K_{\mathrm{MD}}, K_{\mathrm{SD}}$ and $K_{\mathrm{AD}}$ are constants that account for the displacement of the partitioning equilibria by the organic solvent. The $K_{\mathrm{SD}}$ coefficient has been found to be significant only for highly hydrophobic compounds. When this is not the case, Eq. (5) can be simplified to:

$$
\frac{1}{k}=\frac{1}{K_{\mathrm{AS}}}\left(1+K_{\mathrm{AD}} \varphi\right)+\frac{K_{\mathrm{AM}}}{K_{\mathrm{AS}}}\left(1+K_{\mathrm{MD}} \varphi\right)[M]=c_{0}+c_{1} \varphi+c_{2}[M]+c_{12} \varphi[M]
$$

where $c_{0}, c_{1}, c_{11}$ and $c_{12}$ are again regression coefficients, all with positives values. A similar approach has been found to be valid for the submicellar modes. The following model describes accurately the retention in HSLC [14,15]:

$$
\begin{aligned}
\frac{1}{k} & =\frac{1}{K_{\mathrm{AS}}}\left(1+K_{\mathrm{AD}} \varphi\right)+\frac{K_{\varphi}}{K_{\mathrm{AS}}}\left(1+K_{\mathrm{AD}} \varphi\right) \varphi^{2}+\frac{K_{\mathrm{AM}}^{\mathrm{HSC}}}{K_{\mathrm{AS}}}\left(1+K_{\mathrm{MD}} \varphi\right)[S]= \\
& =c_{0}+c_{1} \varphi+c_{11} \varphi^{2}+c_{111} \varphi^{3}+c_{2}[S]+c_{12} \varphi[S]
\end{aligned}
$$

where the quadratic and cubic terms in $\varphi$ were added to account for the larger role of the organic solvent in the mobile phase; $K_{\mathrm{AM}}$ and $K_{\mathrm{MD}}$ refer to the interaction of solutes with surfactant monomers instead of micelles, $K_{\varphi}$ is a regression coefficient similar to $c_{11}$ in Eq. (3), and $K_{\mathrm{AM}}^{\mathrm{HSC}}$ describes the partitioning between bulk water and the free monomers. A simplified equation is:

$$
\frac{1}{k}=\frac{1}{K_{\mathrm{AS}}}\left(1+K_{\mathrm{AD}} \varphi\right)+\frac{K_{\varphi}}{K_{\mathrm{AS}}} \varphi^{2}+\frac{K_{\mathrm{AM}}^{\mathrm{HSC}}}{K_{\mathrm{AS}}}\left(1+K_{\mathrm{MD}} \varphi\right)[S]=c_{0}+c_{1} \varphi+c_{11} \varphi^{2}+c_{2}[S]+c_{12} \varphi[S]
$$

The elution behaviour of $10 \beta$-blockers was studied using a Kromasil C18 column, in wide ranges of SDS $(0.075-0.15 \mathrm{M})$ and acetonitrile $(5-50 \%(\mathrm{v} / \mathrm{v}))$, involving the micellar and high submicellar regions [14]. Eqs. (6) and (8) yielded excellent descriptions of the retention in each region, respectively. When the whole search space was considered, the use of specific models for different regions of the factor space complicated the exploration of the 
optimal experimental conditions. Therefore, a model that fitted satisfactorily the elution behaviour in the whole domain (5-50\%) was proposed (Eq. (7)). However, as expected, the quality of the predictions was better when the domain was divided in the micellar and submicellar regions, using specific models (Eqs. (6) and (8)) [15].

\section{Peak shape for basic compounds}

In conventional RPLC, the stationary phase is scarcely modified when the organic solvent content is changed, at least in narrow composition ranges $[21,50]$. In the surfactant-mediated modes, the stationary phase nature changes significantly with the mobile phase composition, since the surfactant monomers associate to the alkyl-bonded chains (Fig. 1). This coating is narrowed upon addition of an organic solvent, which dissolves the surfactant [7].

As commented in Section 4.3, the peak width and symmetry of basic compounds eluted from alkyl-bonded silica, which depend on the mass transfer kinetics, are excellent tools to probe the surfactant layer on the stationary phase in an SDS/organic solvent system [2,27]. The undesirable interaction of positively charged basic compounds with ionized silanols on such stationary phases is a slow process, which results in poor peak shape (broad and asymmetrical peaks). This makes the analysis of these compounds by conventional RPLC problematic. Under submicellar conditions at low surfactant concentration, the adsorbed SDS monomers form a layer that masks efficiently the silanols on the siliceous support, preventing their interaction with the basic compounds. These instead interact with the anionic sulphate group in the surfactant through an ion-exchange mechanism, which seems to be a fast process. The result is a large increase in column efficiency. At high organic solvent contents, the surfactant will be significantly desorbed, favouring again solute penetration and interaction with the buried silanols: the efficiency deteriorates. 
In the micellar mode without organic solvent, where the concentration of surfactant is larger, the efficiency deteriorates due to the high carbon loading in the thicker SDS layer, which gives rise to poor stationary-phase diffusion [24]. The MLC literature contains numerous comments on the reduced efficiency for compounds of different nature eluted with micellar mobile phases containing exclusively a surfactant (either ionic or non-ionic). Organic solvent addition and temperature raise have been given as solutions to decrease the amount of adsorbed surfactant, and improve the efficiency [1,8]. However, at increasing solvent contents, after reaching a plateau, further surfactant desorption will allow the interaction of basic drugs with the unmasked ionized silanols on the C18 stationary phase, yielding again poor efficiency and skewness.

In HSLC (obtained at high surfactant and organic solvent contents), the efficiency has been observed to be similar to that in IPC, and often larger than in the micellar mode. This is apparently due to the thinner SDS layer, which masks the silanols allowing sufficiently large solute diffusion. Wherever enough surfactant coats the stationary phase (up to 60\% methanol, 40\% ethanol, 35\% 1-propanol, and 50\% acetonitrile), the efficiency will be high.

In the literature, the comparison of peak shapes is usually made based on the individual or mean values of the efficiencies (or widths) and asymmetries, for several compounds. A main problem associated to the use of these mean values is that different conditions and compounds give rise to different elution strengths, and consequently, the retention time ranges change. However, owing to the extra-column broadening contribution to the global variance (which becomes more significant as retention decreases), only the efficiencies for peaks eluting at similar retention times should be compared. The linear relationships between the left and right half-widths and the retention times (which have been called peak half-width plots) allow a fairer comparison of the behaviour under different conditions [51,52]: 
$A=m_{\mathrm{A}} t_{\mathrm{R}}+A_{0}$

$$
B=m_{\mathrm{B}} t_{\mathrm{R}}+B_{0}
$$

where $A$ and $B$ are the peak half-widths, which are conveniently measured at $10 \%$ peak height), $t_{\mathrm{R}}$ is the retention time, $m_{\mathrm{A}}$ and $m_{\mathrm{B}}$ are the slopes of the linear correlations, and $A_{0}$ and $B_{0}$ the corresponding intercepts. Note that the peak half-width plots are indeed parabolic, but this is only evident for wide ranges of retention times.

Eqs. (9) and (10) allow the prediction of the peak widths and asymmetries at different retention times, and provide useful information to characterize the column performance: the sum of slopes $\left(m_{\mathrm{A}}+m_{\mathrm{B}}\right)$ represents the peak broadening rate, and the ratio $m_{\mathrm{B}} / m_{\mathrm{A}}$ the peak asymmetry inside the chromatographic column. In a comprehensive study, the behaviour of acetonitrile and the alcohols methanol, ethanol and 1-propanol on the peak shape of a set of basic compounds ( $\beta$-blockers) eluted with hydro-organic, micellar and submicellar mobile phases was examined, using conventional silica-based columns [52]. The following observations were made:

(i) The peak broadening rate $\left(m_{\mathrm{A}}+m_{\mathrm{B}}\right)$ was significantly smaller in the surfactant-mediated modes compared to the hydro-organic mode.

(ii) In the hydro-organic mode, peak deformation was significant. The lines diverged with $m_{\mathrm{B}} / m_{\mathrm{A}}$ usually in the range $2.5-5$, corresponding to tailing peaks.

(iii) The peaks were nearly symmetrical in the presence of surfactant and organic solvent (i.e. $m_{\mathrm{B}} / m_{\mathrm{A}} \approx 1$ ). The peak half-width plots almost coincided, being parallel, or diverging/converging only slightly.

(iv) The best peaks for $\beta$-blockers, in the presence of SDS, were obtained with acetonitrile (compared to ethanol and 1-propanol, which behaved similarly). This was explained by a larger reduction in the adsorbed surfactant layer on the C18 column [15,27]. It should be noted that the peak broadening rate in acetonitrile-water mixtures was also smaller (7-8\% 
against $8-15 \%$ for propanol) [52]. With a Kromasil C18 column and acetonitrile, the poorest efficiencies were obtained for the hydro-organic mode $(N=800-1700)$. These improved in the micellar mode $(N=1000-3300)$. However, the most outstanding enhancements were observed in the submicellar modes, with $N$ values frequently in the 4000-9000 range [2].

\section{Separation performance}

\subsection{Surfactant elution strength}

As commented, the addition of a low concentration of surfactant into a conventional mobile phase in RPLC alters the stationary phase surface and the partitioning behaviour of analytes. The excess surfactant is dissolved in the hydro-organic mobile phase as free monomers, associated in small clusters or forming micelles. These entities and the organic solvent molecules are responsible of the elution. The magnitude of the effect can be modulated by varying both the nature and concentration of the surfactant and organic solvent. In the pioneering work by Li and Fritz on the use of surfactants at concentrations above their CMC in aqueous solution but without micelle formation, surfactants with different elution strengths were investigated in the presence of $60 \%$ acetonitrile [10]. The degree of reduction in the retention times of analytes was determined by the hydrophobic chain length and chemical nature of the surfactant. In the case of non-ionic surfactants (as Tween 60), used to separate a set of alkylphenols, hydrogen bond formation between the hydroxyl groups in the surfactant and those in the phenols probably took place in addition to hydrophobic interactions between the hydrophobic parts of analytes and Tween 60. Later, the authors studied mobile phases containing mixtures of two surfactants in the presence of $40-60 \%$ acetonitrile, where no co-micellization was expected [12]. The elution strength was increased in the presence of the surfactants. 
In the comprehensive study with $\beta$-blockers described above [27], a significant difference in behaviour was found between IPC and MLC/HSLC, when the concentration of SDS in the mobile phase was increased. In IPC, the retention was progressively larger, since the surfactant-coating was still growing and the amount of surfactant monomers in the mobile phase was rather low. In MLC/HSLC, the effect of the surfactant was opposite to IPC (the retention decreased with the addition of more surfactant), as a consequence of the additional interactions with micelles or free surfactant monomers in the mobile phase. In MLC, the surfactant coating reaches or is next to saturation, and the amount of micelles in the mobile phase (to which the cationic solutes are strongly associated) increases. In HSLC, the surfactant coating has been reduced significantly with regard to MLC, and the added surfactant monomers (to which the cationic solutes are also associated) remain free in the mobile phase. Both micelles (in MLC) and free surfactant monomers (in HSLC) increase the solubilization capability of the mobile phase, and accordingly, the elution strength.

A common topic in the MLC literature is the role of micelles in the chromatographic behaviour. Certainly, micelles increase the solubility of analytes, and contribute to their desorption from the stationary phase, with an elution strength often larger than that of the organic solvent. Thus, for $\beta$-blockers in MLC and HSLC, the surfactant (SDS) was significantly stronger than short chain alcohols and acetonitrile. The reason for this behaviour is the electrostatic association of the cationic drugs with the anionic micelles or surfactant monomers, which is stronger than the hydrophobic association with organic solvent molecules.

\subsection{Organic solvent strength}

In the surfactant-mediated modes, the organic solvent is seen as a secondary modifier, which can affect the micelle nature and displace the analyte partition equilibrium towards the 
bulk mobile phase. However, the role of the organic solvent is not far from that in a hydro-organic mixture. The loss of protagonism can be explained by its association with the micelles or surfactant monomers, which decreases its capability to interact with analytes. Since the stabilization with an organized structure (as the micelles) is stronger, disruption of micelles at high concentration of organic solvent is translated into a significant increase in the elution strength, which becomes similar to that observed with a hydro-organic mobile phase in the absence of surfactant. Thus, for example, a significant increase in the elution strength of acetonitrile (for 0.075-0.15 M SDS) was observed at increasing organic solvent contents: the slopes of the plots for $30-50 \%$ acetonitrile (the high submicellar region) were larger than for 5-20\% acetonitrile (the micellar region), with a transition region in the range 20-30\% acetonitrile (Fig. 3) [2,14]. In the transition region, two effects happened that affected the retention: the micelles were being perturbed and the surfactant monomers covering the stationary phase desorbed (both significantly) by the organic solvent. However, no discontinuity was observed between the micellar and submicellar modes, at constant surfactant concentration.

Non-linear dependences were achieved for $\log k$ versus $\varphi$ (Eq. (3)) in sufficiently large concentration ranges, being the curves concave for the hydro-organic (without surfactant) and IPC modes, and convex for MLC/HSLC (as indicated above, the elution strength was progressively larger) (Fig. 3) [27]. At sufficiently large methanol and acetonitrile contents, the slopes of the curves obtained with SDS were similar to those without surfactant and a smaller amount of organic solvent. Thus, the slopes for $0.075 \mathrm{M} \mathrm{SDS} / 50-60 \%$ methanol and 40-50\% methanol-water, on the one hand, and for $0.075 \mathrm{M} \mathrm{SDS} / 30-50 \%$ acetonitrile and 10-25\% acetonitrile-water, on the other, were similar. Ethanol and 1-propanol were still stronger in the hydro-organic mobile phases, for the assayed ranges. Among the alcohols, only 1-propanol allowed the inspection of a wide range of experimental conditions. The feasible experimental 
domain was narrower for ethanol and methanol, especially for the latter, owing to its smaller elution strength.

There is an extensive discussion on the association of solutes with micelles. However, there is little information about the effect of micelles or surfactant monomers on the organic solvent molecules that affect their behaviour as modifiers. Short-chain alcohols (i.e. methanol to 1-propanol) have a small penetration capability into SDS micelles. The binding constants (expressed as mole fraction ratio of organic solvent per surfactant molecule) are: $0.4,1.1$, and 3.5 for methanol, ethanol, and 1-propanol, respectively, at $25^{\circ} \mathrm{C}[49,53]$. These values correlate with the logarithm of the octanol-water partition coefficient of the solvents $\left(\log P_{\mathrm{o} / \mathrm{w}}=0.18,0.48\right.$, and 2.2, respectively [54]). $\log P_{\mathrm{o} / \mathrm{w}}$ for acetonitrile is similar to that for ethanol (0.46). However, the effect of acetonitrile on the CMC of SDS is similar to that of methanol (i.e. the CMC increases at increasing concentration of organic solvent), and opposite to the effect of ethanol and 1-propanol (i.e. the CMC decreases) [26]. The relatively strong association of 1-propanol to the SDS micelles can explain the smaller elution strength below 15-25\% 1-propanol for 0.02 and 0.04 M SDS.

The weak elution strength of methanol, ethanol and acetonitrile force the use of higher concentrations of surfactant. Also, with methanol the mobile phases should contain a large amount of organic solvent to achieve convenient retention times, reaching often submicellar conditions. As commented, the retention times of positively charged basic compounds in the surfactant-mediated chromatographic modes are longer with regard to hydro-organic RPLC, owing to their strong attraction to the stationary phase. This was found for $\beta$-blockers at least up to $50 \%$ acetonitrile [2,27]. Owing to the increased retention, the surfactant-mediated modes allowed wider concentration ranges for the organic solvents than the hydro-organic mode, except for methanol $[15,27]$. In general, the concentration ranges for both surfactant and organic solvent should be selected to achieve enough retention for the most polar 
compounds, and not excessive retention for the most apolar ones. The pump back-pressure at increasing concentration of both modifiers limits also their maximal content in the mobile phase.

\subsection{Gradient elution and accordion effect}

Gradient elution is commonly used to separate relatively complex mixtures of compounds in wide polarity ranges. In conventional RPLC, a gradient of organic solvent is generally applied to decrease the analysis time, since an increasing amount of organic solvent would result in a stronger eluent. IPC is less suitable for gradient elution, due to the strong dependence of the adsorbed amount of surfactant with the concentration of organic solvent in the mobile phase. This can make re-equilibration after the end of each run lengthy and tedious. In MLC, gradient elution by varying the surfactant is favoured because at moderate surfactant concentration, the composition of the stationary phase is independent of the concentration of micelles in the mobile phase during the gradient: the surfactant layer on the stationary phase depends only on the free surfactant concentration and a change in the total concentration serves only to change the concentration of micelles $[55,56]$. Therefore, the only re-equilibration process necessary before the next gradient run is flushing the chromatographic system with the initial mobile phase. However, this rapid gradient capability is not universal: significant surfactant adsorption is observed above the CMC for non-ionic surfactants.

Gradient elution with organic solvent in MLC is, however, problematic, since along the gradient the organic solvent desorbs the surfactant. Going back to the initial conditions should require a long equilibration time. In HSLC, the surfactant layer is significantly thinner. Therefore, gradient elution by varying the organic solvent concentration is possible [10]. Since no surface adsorption is taking place, gradient elution can be very fast because no 
re-equilibration of the column is required. In a recent report, a gradient method was designed, starting with a micellar eluent with a low concentration of butanol [57]. This allowed direct injection of plasma due to the solubilisation of proteins. After eluting the proteins, the concentration of butanol was increased (obtaining HSLC conditions) to reduce the analyte retention time and enhance the performance. Consequently, the authors demonstrated that the transition from MLC to HSLC has a potential interest.

It appears, however, that the addition of a surfactant to the mobile phase at fixed concentration provides often similar benefits to conventional solvent gradient elution. The retention of all analytes decreases in the presence of surfactant but to different degrees. The surfactant complex late-eluting analytes (larger and more hydrophobic) more strongly, and thereby, reduces their retention times by a larger percentage than the retention times of earlier analytes. Consequently, the sample peaks are rather evenly distributed in the chromatogram. This is a noticeable gradient elution feature, although here only isocratic elution with a hybrid surfactant/organic solvent eluent is used [58]. This outstanding feature is found in both MLC and HSLC [1,10]. In these chromatographic modes, real gradient elution is therefore less necessary.

\subsection{Selectivity and resolution}

The main reason to modify both or either stationary phase and mobile phase is the improvement in analysis time and selectivity. These depend on the relative interactions of solutes with both phases. The additional interactions that take place inside a chromatographic column, in the presence of a surfactant, give rise to changes in the absolute and relative retention, and for some compounds to better peak profiles. Not only the elution strength but also the elution order and resolution performance (which depends on the selectivity and peak shape) are changed. This may enhance the performance with respect to conventional RPLC. 
By choice of the nature and/or concentration of the modifiers (surfactant and organic solvent), the solvent strength and selectivity can be varied according to actual needs. The effect is similar or enhanced with respect to adjusting the composition of a ternary mobile phase comprised of water and two organic solvents in RPLC. Anyway, it offers another possibility for fine-tuning the selectivity and enhancing the chromatographic performance.

The selectivity in submicellar mobile phases may differ significantly from the selectivity in hydro-organic or micellar mobile phases. The analyst can take advantage of this to improve the resolution in specific separation problems. HSLC can, therefore, be considered as a complement to MLC. Excellent separations with a variety of surfactants (such as Brij 30, THPA, DOSS and Tween 60), compared to conventional RPLC, have been reported for mixtures containing compounds with various polarities and functionalities. Thus, the presence of a surfactant in hydro-organic mobile phases has been shown to greatly improve the separation of alkylbenzenes, polycyclic aromatic hydrocarbons, alkylphenols, and some other aromatic compounds [11-13]. Compared with separations obtained using hydro-organic mixtures, shorter retention times and sharper peaks were obtained. In some cases, the transition from a hybrid micellar to a submicellar system did not change the separation selectivity, while the analysis time decreased significantly.

Li and Fritz made a comprehensive study, where the HSLC mode was compared with the hydro-organic mode for several surfactants [10]. Some examples of separation are the following:

(i) Seven alkylphenols were completely resolved with $60 \%$ acetonitrile in ca. $22 \mathrm{~min}$. When $50 \mathrm{mM}$ Pluronic L-31 was added to the mobile phase, baseline resolution was still possible requiring only $14 \mathrm{~min}$ (Fig. 4).

(ii) The separation of six aromatic compounds was also complete with $60 \%$ acetonitrile, but took more than 54 min to elute all analytes from the column, because of the strong 
interactions between the highly hydrophobic analytes and the stationary phase (Fig. 5a). When $40 \mathrm{mM}$ Tween 60 (which contains one saturated C17 hydrocarbon chain) was added, the same separation took only about 10 min to finish and yielded much sharper peaks (Fig. 5b).

(iii) When THPA (which contains four saturated C7 hydrocarbon chains) was added to 60\% acetonitrile, a similar effect was observed, but to a lesser degree due to the weaker hydrophobic interaction between these compounds and THPA (Fig. 5c).

The selectivity is traditionally measured through the ratio of the retention factors (i.e. relative retention, called the "selectivity factor") for selected pairs of probe compounds, eluted under specific conditions. The probe compounds are assumed to measure different properties, such as column hydrophobicity, silanol activity, steric hindrance, hydrogen bonding capacity and ion-exchange capability [59]. However, although the conclusions about the hydrophobicity generally agree between the tests, those for other properties differ. Also, it should be noted that the selectivity changes with mobile phase composition. It is, thus, possible that two chromatographic systems show similar for a given composition region and differ extremely for another [60].

As commented, the anionic surfactant SDS adsorbed on the stationary phase increases the retention and improves the peak shape of basic compounds $[14,15]$. This extends the separation space, giving rise to high resolution in wide concentration ranges of both surfactant and organic solvent. A comprehensive description of the selectivity aimed to compare the potentiality of MLC and HSLC with SDS, against conventional RPLC, was performed for mixtures of $\beta$-blockers (Fig. 2). In order to compare the selectivity in different conditions, the retention times of the $\beta$-blockers using different organic solvents (methanol, ethanol, 1-propanol and acetonitrile) were regressed each other, at varying mobile phase composition [15]. The correlation coefficient was used as a descriptor of the similarity between the peak 
distribution (selectivity) of the systems. The addition of surfactant was observed to yield significant changes in the selectivity. In the hybrid systems, different organic solvents gave rise to different selectivities, but the similarities increased at increasing concentration of organic solvent, especially for ethanol and 1-propanol. Methanol and ethanol were similar in selectivity, in a wide composition range. HSLC with acetonitrile appeared as the most promising mode, as it allowed full resolution of the $\beta$-blockers in practical times, while these were unresolved or highly retained in the other RPLC modes (Fig. 2) [15]. Ethanol also provided good separation performance, significantly improved with respect to methanol and 1-propanol. In contrast, the hydro-organic mode with acetonitrile or any of the short-chain alcohols could not succeed with the separation of the $\beta$-blockers, owing to the poorer selectivity and wider peaks.

Surprisingly, trial-and-error optimization strategies are still often applied in the optimization of the separation conditions in RPLC, instead of the more reliable interpretive strategies (based on the description of the retention behaviour of analytes) [61,62]. These have the advantage of allowing a comprehensive examination of the changes in the chromatograms of individual solutes, or mixtures of two or more solutes, making the detailed exploration of the resolution possible, which is especially valuable when two modifiers should be optimized. The mobile phase offering maximal resolution, or at least satisfactory resolution in an adequate analysis time, or with a smaller amount of modifier in the mobile phase is thus facilitated. A software package commercialised in 2000 for MLC can be also useful for developing HSLC methodologies [63]. 
693

694

695

696

697

\section{Conclusions}

Thirty years ago, people working in RPLC using surfactants as additives (an IPC approach) avoided concentrations where micelles could be formed. They were only interested in modifying the stationary phase surface with monomers of surfactant. In 1980, Armstrong and Henry demonstrated that the presence of micelles in the mobile phase cooperated to solute elution, with interesting implications in the selectivity [5]. The total production of scientific reports in MLC up-to-date amounts to several hundreds. Many authors have demonstrated that the technique has several advantages regarding its large versatility produced by the interaction of solutes with different surfactants and organic solvents, the direct injection of physiological fluids which avoids the tedious sample pre-treatment required in conventional RPLC, the suppression of peak tailing for basic drugs, and the analysis of samples containing compounds in a wide range of polarities using isocratic elution, among others $[1,8]$. MLC requires the addition of an organic solvent to reduce the retention times and enhance the peak shape. In order to preserve the micelles, analysts working in MLC avoid high amounts of organic solvent in the mobile phase. Surprisingly, in some reports this seemed to be ignored, since authors claiming to work in MLC employed mobile phase compositions where micelles cannot be formed. The results were highly satisfactory, which demonstrates that there is no reason to neglect the potentiality of mobile phases containing surfactant monomers instead of micelles.

There can be some concern about considering that the new conditions give rise to a particular RPLC mode. The technique could be classified as a particular case of IPC or submicellar liquid chromatography, without delimiting a clear boundary. A superficial look would indicate that the only difference with the classical IPC is that the surfactant concentration is high and the organic solvent content might be larger than conventionally used. The new submicellar mode can be also considered as a bridge between MLC and 
conventional RPLC: the concentration of surfactant is similar to that used in MLC, but the high concentration of organic solvent does not allow the formation of micelles.

Three research groups have investigated this chromatographic mode in the last 15 years, with regard to MLC and conventional RPLC: Li and Fritz [10,12], Jandera and Fischer [13], and recently, Ruiz-Ángel et al. [2,14,15,27,52]. These authors demonstrated that the submicellar RPLC chromatographic mode at high concentration of surfactant, which was abbreviated as high submicellar liquid chromatography merits some attention, since it offers a better separation window than conventional hydro-organic mobile phases and superior separation efficiency compared to MLC, for the analysis of aromatic compounds and basic drugs. The combination of improved peak shape, larger selectivity, smaller analysis times (due to the addition of a higher amount of organic solvent), and smaller range in retention among compounds of extreme polarity, leads to the logical observation that more compounds can be resolved in one run using isocratic elution. The result is a chromatographic mode, which achieves in practical times separations of compounds unresolved, or highly retained with other RPLC modes.

The consumption of organic solvent in HSLC is higher with respect to MLC, which can be considered as a drawback. However, in the presence of surfactant, the risk of evaporation decreases due to the solubilisation of the organic solvent molecules by the surfactant. This facilitates mobile phase recycling. It should be finally said that the addition of surfactants to the mobile phase complicates the use of mass spectrometric detection, and may add noise or a background signal to UV detection.

Acknowledgments. This work was supported by Project CTQ2010-16010/BQU (Ministerio de Ciencia e Innovación of Spain), and FEDER funds. 


\section{References}

[1] Berthod, A., and García-Álvarez-Coque, M.C. (2000) Micellar Liquid Chromatography; Marcel Dekker, New York, USA.

[2] Ruiz-Ángel, M.J., Torres-Lapasió, J.R., García-Álvarez-Coque, M.C., and Carda-Broch, S. (2008) Retention mechanisms for basic drugs in the submicellar and micellar reversed-phase liquid chromatographic modes. Anal. Chem., 80: 9705-9713.

[3] Ruiz-Ángel, M.J., Carda-Broch, S., Torres-Lapasió, J.R., and García-Álvarez-Coque, M.C. (2009) Retention mechanisms in micellar liquid chromatography. J. Chromatogr. A, 1216: 1798-1814.

[4] Berthod, A., Girard, I., and Gonnet, C. (1986) Micellar liquid chromatography, adsorption isotherms of two ionic surfactants on five stationary phases. Anal. Chem., 58: $1356-1358$.

[5] Armstrong, D.W., and Henry, S.J. (1980) Use of an Aqueous Micellar Mobile Phase for Separation of Phenols and Polynuclear Aromatic Hydrocarbons via HPLC. J. Liq. Chromatogr., 3: 657-662.

[6] Dorsey, J.G., DeEchegaray, M.T., and Landy, J.S. (1983) Efficiency enhancement in micellar liquid chromatography. Anal. Chem., 55: 924-928.

[7] López-Grío, S., García-Álvarez-Coque, M.C., Hinze, W.L., Quina, F.H., and Berthod, A. (2000) Effect of a Variety of Organic Additives on Retention and Efficiency in Micellar Liquid Chromatography. Anal. Chem., 72: 4826-4835.

[8] Ruiz-Ángel, M.J., García-Álvarez-Coque, M.C., and Berthod, A. (2009) New Insights and Recent Developments in Micellar Liquid Chromatography. Sep. Purif. Rev., 38: 4596.

[9] Zana, R. (1995) Aqueous surfactant-alcohol systems: A review. Adv. Colloid Interface Sci., 57: 1-64. 
[10] Li, X., and Fritz, J.S. (1996) Novel additives for the separation of organic compounds by high-performance liquid chromatography. J. Chromatogr. A, 728: 235-247.

[11] Fischer, J., and Jandera, P. (1996) Chromatographic behaviour in reversed-phase highperformance liquid chromatography with micellar and submicellar mobile phases: effects of the organic modifier. J. Chromatogr. B, 681:3-19.

[12] Li, X. and Fritz, J.S. (1996) Mixed surfactants as mobile phase additives for the separation of organic compounds by HPLC. Anal. Chem., 68: 4481-4484.

[13] Jandera, P., Fischer, J., and Effenberger, H. (1998) Characterisation of retention in micellar high-performance liquid chromatography and in micellar electrokinetic chromatography using lipophilicity and polarity indices. J. Chromatogr. A, 807: 57-70.

[14] Ruiz-Ángel, M.J., Torres-Lapasió, J.R., García-Álvarez-Coque, M.C., and Carda-Broch, S. (2009) Submicellar and micellar reversed-phase liquid chromatographic modes applied to the separation of $\beta$-blockers. J. Chromatogr. A, 1216: 3199.

[15] Ruiz-Ángel, M.J., Torres-Lapasió, J.R., Carda-Broch, S., and García-Álvarez-Coque, M.C. (2010) Performance of short-chain alcohols versus acetonitrile in the surfactantmediated reversed-phase liquid chromatographic separation of $\beta$-blockers. J. Chromatogr. A, 1217: 7090-7099.

[16] Cecchi, T. And Passamonti, P. (2009) Retention mechanism for ion-pair chromatography with chaotropic reagents. J. Chromatogr. A, 1216: 1789-1797.

[17] Torres-Lapasió, J.R., Villanueva-Camañas, R.M., Sanchis-Mallols, J.M., MedinaHernández, M.J., and García-Álvarez-Coque, M.C. (1993) Modelling of the retention behaviour of solutes in micellar liquid chromatography with organic modifiers. $J$. Chromatogr. A, 639: 87-96. 
791

792

793

794

[18] López-Grío, S., Baeza-Baeza, J.J., and García-Álvarez-Coque, M.C. (2001) Evaluation of the elution strength of the surfactant and organic solvent in hybrid micellar mobile phases. J. Liq. Chromatogr. Rel. Technol., 24: 2765-2783.

[19] Lavine, B.L., Cooper, W.T., He, Y., Hendayana, S., Han, J.H., and Tetreault, (1994) J. Solid-State ${ }^{13} \mathrm{C}$ NMR Studies of Ionic Surfactants Adsorbed on C-18 and C-8 Silicas: Implications for Micellar Liquid Chromatography. J. Colloid Interface Sci., 165: $497-$ 504.

[20] Morris, J., and Fritz, J.S. (1994) Eluent Modifiers for the Liquid Chromatographic Separation of Carboxylic Acids Using Conductivity Detection. Anal. Chem., 66: 23902396.

[21] Montgomery Jr., M.E., and Wirth M.J. (1994) Spectroscopic Study of the Molecular Basis of Wetting of a C18 Surface by Long-Chain n-Alcohols. Anal. Chem., 66: 680684.

[22] Hansen, S.H., Helboe, P., and Thomsen, M. (1991) Bare silica, dynamically modified with long-chain quaternary ammonium ions-the technique of choice for more reproducible selectivity in reversed-phase high-performance liquid chromatography. $J$. Chromatogr., 544: 53-76.

[23] Lavine, B.K., Hendayana, S., Cooper, W.T., and He, Y. (1999) Surfactant-Bonded Phase Associations in Micellar Reversed Phase Liquid Chromatography. ACS Symp. Ser., 740: 290-313.

[24] Borgerding, M.F., Hinze, W.L., Stafford, L.D., Fulp Jr., G.W., and Hamlin Jr., W.C. (1989) Investigations of stationary phase modification by the mobile phase surfactant in micellar liquid chromatography. Anal. Chem., 61 (1989) 1353-1358.

[25] Mittal, K.L. (Editor) (1979) Micellization, Solubilization and Microemulsions, Plenum Press, New York, USA, Vol. 1. 
[26] López-Grío, S., Baeza-Baeza, J.J., and García-Álvarez-Coque, M.C. (1998) Influence of the addition of modifiers on solute-micelle interaction in hybrid micellar liquid chromatography. Chromatographia, 48: 655-663.

[27] Ruiz-Ángel, M.J., Carda-Broch, S., and García-Álvarez-Coque, M.C. (2010) Effect of short-chain alcohols on surfactant-mediated reversed-phase liquid chromatographic systems. J. Chromatogr. A, 1217: 7082-7089.

[28] Posluszny, J.V., and Weinberger, R. (1988) Determination of drug substances in biological fluids by direct injection multidimensional liquid chromatography with a micellar cleanup and reversed-phase chromatography. Anal. Chem., 60: 1953-1958.

[29] Li, Y.M., Chen, L.R., and Qu, Y. (1993) Use of Micellar Mobile Phases and an HPLC Column Switching System for Direct Injection Determination of Urinary Free Cortisol. J. Liq. Chromatogr., 16: 2583-2594.

[30] Strege, M.A., and Lagu, A.L. (1995) Analysis of recombinant human growth hormone in Escherichia coli fermentation broth by micellar high-performance liquid chromatography. J. Chromatogr. A, 705: 155-161.

[31] Aparicio, A., San-Andrés, M.P., and Vera, S. (2000) Separation and determination of phenolic antioxidants by HPLC with surfactant/n-propanol mobile phases. J. High Resolut. Chromatogr., 23: 324-328.

[32] Paleologos, E.K., Chytiri, S.D., Savvaidis, I.N., and Kontominas, M.G. (2003) Determination of biogenic amines as their benzoyl derivatives after cloud point extraction with micellar liquid chromatographic separation. J. Chromatogr. A, 1010: 217-224.

[33] Paleologos, E.K., and Kontominas, M.G. (2004) On-Line Solid-Phase Extraction with Surfactant Accelerated On-Column Derivatization and Micellar Liquid 
Chromatographic Separation as a Tool for the Determination of Biogenic Amines in Various Food Substrates. Anal. Chem., 76: 1289-1294.

[34] Kulikov, A.U., and Verushkin, A.G. (2004) Development and Validation of a Micellar Liquid Chromatographic Method with UV Detection for Determination of Azithromycin in Tablets and Capsules. Chromatographia, 60: 33-38.

[35] del Olmo, M., Garballo, A., Nimer, M., López, I., De Ferrer, J.A., and Vílchez, J.L. (2004) Development of Methodologies for Different Degrees of Resolution of Linear Alkylbenzene Sulfonates in Groundwater and Wastewater by Liquid Chromatography Using Sodium Dodecyl Sulphate. Chromatographia, 60: 157-164.

[36] Jandera, P., and Fischer, J. (1996) Chromatographic behaviour in reversed-phase highperformance liquid chromatography with micellar and submicellar mobile phases. $J$. Chromatogr. A, 728 (1996) 279-298.

[37] Romani, A.P., Gehlen, M.H., Lima, G.A.R., and Quina, F.H. (2001) The Change in the Properties of Sodium Dodecyl Sulfate Micelles upon Addition of Isomeric and Unsaturated Short-Chain Alcohols Probed by Photophysical Methods. J. Colloid Interface Sci., 240: 335-339.

\section{[38] A. Berthed, I. Girard, C. Gennet, C. Anal. Chem. 58(1986) 1356.}

[39] Berthod, A., Girard, I., and Gonnet, C. (1986) Additive effects on surfactant adsorption and ionic solute retention in micellar liquid chromatography. Anal. Chem., 58: 13621367.

[40] Berthod, A., and Roussel, A. (1988) The rôle of the stationary phase in micellar liquid chromatography: Adsorption and efficiency. J. Chromatogr., 449: 349-360.

[41] Scott, R.P.W., and Simpson, C.F. (1980) Solute-solvent interactions on the surface of reversed phases: I. Stationary phase interactions and their dependence on bonding characteristics. J. Chromatogr., 197: 11-20. 
[42] Borgerding, M.F., Williams Jr., R.L., Hinze, W.L., and Quina, F.H. (1989) New Perspectives in Micellar Liquid Chromatography. J. Liq. Chromatogr. 12: 1367-1406.

[43] Armstrong, D.W., and Stine, G.Y. (1983) Evaluation of Partition Coefficients to Micelles and Cyclodextrins via Planar Chromatography. J. Am. Chem. Soc., 105: 29622964.

[44] Poppe, H. (1993) Distribution isotherms in reversed-phase systems. J. Chromatogr. A, 656: 19-36.

[45] Nawrocki, J. (1997) The silanol group and its role in liquid chromatography. J. Chromatogr. A, 779: 29-71.

[46] Berthod, A., Borgerding, M.F., and Hinze, W.L. (1991) Investigation of the causes of reduced efficiency in micellar liquid chromatography. J. Chromatogr., 556: 263-275.

[47] Armstrong, D.W., and Nome, F. (1981) Partitioning behavior of solutes eluted with micellar mobile phases in liquid chromatography. Anal. Chem., 53: 1662-1666.

[48] García-Álvarez-Coque, M.C., Torres-Lapasió, J.R., Baeza-Baeza, J.J. (1997) Modelling of retention behaviour of solutes in micellar liquid chromatography. J. Chromatogr. A, 780: $129-148$.

[49] Borgerding, M.F., Quina, F.H., Hinze, W.L., Bowermaster, J., and McNair, H.M. (1988) Investigation of the retention mechanism in nonionic micellar liquid chromatography using an alkylbenzene homologous series. Anal. Chem., 60: 25202527.

[50] Tan, L.Ch., and Carr, P.W. (1998) Study of retention in reversed-phase liquid chromatography using linear solvation energy relationships: II. The mobile phase. $J$. Chromatogr. A, 799: 1-19. 
[51] Baeza-Baeza, J.J., Ruiz-Ángel, M.J., and García-Álvarez-Coque, M.C. (2007) Prediction of peak shape in hydro-organic and micellar-organic liquid chromatography as a function of mobile phase composition. J. Chromatogr. A, 1163: 119-127.

[52] Ruiz-Ángel, M.J., Carda-Broch, S., and García-Álvarez-Coque, M.C. (2010) Peak halfwidth plots to study the effect of organic solvents on the peak performance of basic drugs in micellar liquid chromatography. J. Chromatogr. A, 1217: 1786-1798.

[53] De Lisi, R., and Milioto, S. (1988) Excess enthalpies of solution of some primary and secondary alcohols in sodium dodecylsulfate micellar solutions. J. Solution Chem., 17: 245-265.

[54] Leo, A., Hansch, C., and Elkins, D. (1971) Partition coefficients and their uses. Chem. Rev., 71: 525-616.

[55] Khaledi, M.G., and Dorsey, J.G. (1985) Hydro-organic and micellar gradient elution liquid chromatography with electrochemical detection. Anal. Chem., 57: 2190-2196.

[56] Dorsey, J.G., Khaledi, M.G., Landy, J.S., and Lin, J.L. (1984) Gradient elution micellar liquid chromatography. J. Chromatogr., 316: 183-191.

[57] Nakao, R., Schou, M., and Halldin, Ch. (2012) Direct Plasma Metabolite Analysis of Positron Emission Tomography Radioligands by Micellar Liquid Chromatography with Radiometric Detection. Anal. Chem., 84: 3222-3230.

[58] Ruiz-Ángel, M.J., Caballero, R.D., Simó-Alfonso, E., and García-Álvarez-Coque, M.C. (2002) Micellar liquid chromatography: suitable technique for screening analysis. J. Chromatogr. A, 947: 31-45.

[59] Claessens, H.A., van Straten, M.A., Cramers, C.A., Jesierska, M., and Buszewski, B. (1998) Comparative study of test methods for reversed-phase columns for highperformance liquid chromatography. J. Chromatogr. A, 826: 135-156. 
912 [60] Pous-Torres, S., Torres-Lapasió, J.R., Ruiz-Ángel, M.J., and García-Álvarez-Coque, 913 M.C. (2009) Performance of a Chromolith RP-18e column for the screening of beta914 blockers. J. Sep. Sci., 32: 2841-2853.

915 [61] García-Álvarez-Coque, M.C., Torres-Lapasió, J.R., and Baeza-Baeza, J.J. (2006) 916 Models and objective functions for the optimisation of selectivity in reversed-phase 917 liquid chromatography. Anal. Chim. Acta, 579: 125-145.

918 [62] Torres-Lapasió, J.R., and García-Álvarez-Coque, M.C. (2006) Levels in the interpretive optimisation of selectivity in high-performance liquid chromatography: A magical mystery tour. J. Chromatogr. A, 1120: 308-321.

[63] Torres-Lapasió, J.R. (2000) MICHROM Software, Chromatographic Science Series, Marcel Dekker, New York. 


\section{FIGURE CAPTIONS}

Fig. 1. Three-phase systems in alkyl-bonded silica modified with SDS: low submicellar (a), micellar (b), and high submicellar conditions (c), and in naked silica modified with CTAB: micellar (d). In (a), (b) and (c), acetonitrile was added.

Fig. 2. Top: Two-factor space and mobile phase compositions assayed to screen the properties of the different separation environments, using a C18 Kromasil column. Bottom: Chromatograms for the marked compositions in the two-factor space. Mobile phase compositions: (a) 15\% acetonitrile, (b) $30 \%$ acetonitrile, (c) $10^{-3} \mathrm{M} \mathrm{SDS} / 30 \%$ acetonitrile, (d) $5 \times 10^{-3}$ M SDS/50\% acetonitrile, (e) $0.1125 \mathrm{M} \mathrm{SDS} / 10 \%$ acetonitrile, (f) $0.1125 \mathrm{M}$ SDS/17.5\% acetonitrile, (g) $0.1125 \mathrm{M} \mathrm{SDS} / 25 \%$ acetonitrile, (h) $0.1125 \mathrm{M}$ SDS/35\% acetonitrile, (i) $0.1125 \mathrm{M} \mathrm{SDS} / 45 \%$ acetonitrile. Compounds: (1) atenolol, (2) carteolol, (3) pindolol, (4) timolol, (5) acebutolol, (6) metoprolol, (7) esmolol, (8) celiprolol, (9) oxprenolol, and (10) labetalol. Reproduced with permission from Ref. [14].

Fig. 3. Retention behavior of pindolol eluted with hydro-organic mobile phases (dotted lines), and mobile phases containing 0.075 M SDS and organic solvent at increasing concentration

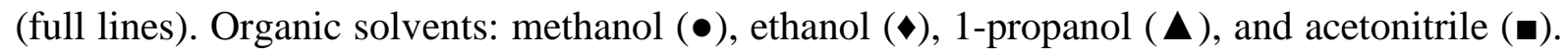
Reproduced with permission from Ref. [27]. 
944 Fig. 4. Chromatographic separation of several alkylphenols on Supelcosil LC-18 (150×4.6 945 mm I.D.) column. Mobile phase: (a) 60\% acetonitrile, and (b) $60 \%$ acetonitrile containing $94650 \mathrm{mM}$ Pluronic L-31. The flow rate was $1 \mathrm{~mL} / \mathrm{min}$, and the peaks were detected at $254 \mathrm{~nm}$.

947 Compounds: $1=$ phenol, $2=p$-cresol, $3=4$-ethylphenol, $4=4-n$-propylphenol, $5=4-n$ 948 butylphenol, $6=4-n$-amylphenol, and $7=4-n$-heptylphenol. Reproduced with permission 949 from Ref. [10].

950

Fig. 5. Chromatographic separation of several aromatic compounds on Supelcosil LC-18 (150×4.6 mm I.D.) column. Mobile phase: (a) $60 \%$ acetonitrile, (b) $60 \%$ acetonitrile containing $40 \mathrm{mM}$ Tween 60 , and (c) $60 \%$ acetonitrile containing $50 \mathrm{mM}$ THPA. The flow rate was $1 \mathrm{~mL} / \mathrm{min}$, and the peaks were detected at $254 \mathrm{~nm}$. Compounds: $1=$ benzene, $2=$ naphthalene, $3=$ anthracene, $4=$ pyrene, $5=$ chrysene, and $6=$ perylene. Reproduced with permission from Ref. [10]. 